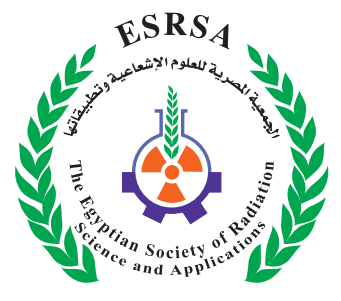

J. Nucl. Tech. Appl. Sci., Vol. 7, PP. 1 : 8 (2019)

\title{
Gonadotropin-Releasing Hormone Antagonist Protects Against Y-Radiation- Induced Female Infertility in Rats
}

Tamim, D.A. ${ }^{1}$; Hamdy, N.M. ${ }^{2}$; Kamal, M.M. ${ }^{2}$ and Nada, A.Sh. ${ }^{1}$

Received: 21/08/2018

Accepted: 30/09/2018

E.mail:daliatamim@yahoo.com

\section{KEYWORDS}

Radiation; Oxidative

Stress; Apoptosis;

Fertility.

\section{ABSTRACT}

Radiotherapy is one of the most common and effective cancer treatments. However, it has a profound impact on ovarian function, leading to premature ovarian failure. With the hope of preserving fertility in cancer survivors, the need for an effective radioprotective therapy is evident. The present study investigated the mechanism of potential radioprotective effect of cetrorelix on radiation-induced ovarian failure and whether cetrorelix can stimulate in vivo follicular development in experimental rats. Immature female rats were either exposed to gamma radiation (3.2 Gy, LD20), once and/or treated with cetrorelix $(0.5 \mathrm{mg} / \mathrm{kg})$, once daily for 10 days before irradiation. Histopathological examination further confirmed the radioprotective efficacy of cetrorelix and its in-vivo effect on ovarian follicles' maturation .Cetrorelix significantly reversed the oxidative stress effects of radiation that was evidenced by increasing in lipid peroxide level and decreasing in glutathione level. In conclusion, cetrorelix showed a radioprotective effect.

1. Drug Radiation Research Department, National Center for Radiation Research and Technology, Atomic Energy Authority, Cairo, Egypt

2. Biochemistry Department, Faculty of Pharmacy, Ain Shams University, Cairo, Egypt 


\section{INTRODUCTION}

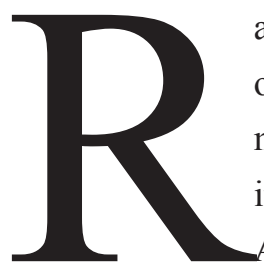

adiotherapy is a well-known cause of ovarian damage that accelerates menopause leading to permanent infertility (Adriaens et al., 2009) Although the damage induced is reversible in sometissues, it is progressive and permanent in the ovary (Stroud et al., 2009).Radiotherapy has a profound impact on ovarian function,leading to depletion of the primordial follicle reserve (Gosden et al., 1997), premature amenorrhea, loss of fertility during or shortly after completion of irradiation (Stroud et al., 2009). Because none of the currently available methods for ovarian protection and fertility preservation guarantees future fertility (Gurgan $\boldsymbol{e t}$ al., 2008), the need for more effective radio protectors has been intensified.

Cetrorelix is a well-known GnRH antagonist which produces immediate and dose-related inhibition of gonadotrophins (FSH,LH) release by competitive blockade of its receptors in the anterior pituitary gland .Furthermore, cetrorelix suppression of gonadotropin secretion can be quickly reversed (Adriaens et al., 2009) . This different pharmacologic mechanism of action makes cetrorelix a more logical choice to use in in-vitro fertilization IVF for the prevention of premature LH surges (Huirne et al., 2007). Collectively, the ovarian radioprotective effect of GnRH antagonist needs to be more cleared. In order to gain a better insight into the mechanism(s) of action, we investigated the influence of cetrorelix on folliculogenesis, ovarian reserve, and oxidative stress.

\section{MATERIALS AND METHODS:}

\section{Drugs and Chemicals:}

GnRH antagonist cetrorelix (Owto Biotech INC., China), glutathione reduced (GSH) and lipid peroxi- dation kits were purchased from (Biodiagnostic Co., Cairo,Egypt).All other chemicals and solvents were of the highest grade commercially available.

\section{Animals}

The study was conducted according to the ethical guidelines (Ain Shams University, Egypt). 80 immature female Sprague-Dawely rats (23 days of age) were obtained from Nile Co. forPharmaceutical and Chemical industries, Egypt. Rats were housed in an air-conditioned atmosphere, at a temperature of $25^{\circ} \mathrm{C}$ with alternatively 12-h light and dark cycles. They were kept on a standard diet and water ad libitum. Standard diet pellets (El-Nasr, Egypt) contained not less than $20 \%$ protein, $5 \%$ fiber, $3.5 \%$ fat, $6.5 \%$ ash and a vitamin mixture.

\section{Premature ovarian failure model}

Whole body gamma-irradiation was carried out using a Cesium $\left({ }^{137} \mathrm{CS}\right)$ source, Gamma Cell-40 biological irradiator, at the National Centre for Radiation Research and Technology (NCRRT), Atomic Energy Authority, Cairo, Egypt. The animals were exposed to a single dose of (3.2 Gy) gamma ray with a dose rate of $0.48 \mathrm{~Gy} / \mathrm{min}$. This dose represents the $\mathrm{LD}_{20}$ according to the study of (Said $\boldsymbol{e t}$ al., 2016). The plastic boxes containing rats were positioned in a chamber fixed to the irradiation equipment.

\section{Experimental Design}

Animals were divided into four groups (20 animals per group) and treated for ten days as follows; the first (Control) and second (Irradiated) groups received vehicle of DMSO and saline at a ratio of 5:95 (0.5 ml/100 g B.W., s.c.) once daily. the second irradiated group exposed to a single dose of (3.2 Gy) whole-body irradiation with gamma ray, on the eleventh day. The third group was given cetrorelix $(0.5 \mathrm{mg} / \mathrm{kg}$, s.c.) dissolved in the vehicle of DMSO and saline once daily. The cetrorelix dose was cho- 
sen according to (Li et al., 2013). The fourth group was administered cetrorelix $(0.5 \mathrm{mg} / \mathrm{kg}$, s.c. $)$ once daily, and then exposed on $11^{\text {th }}$ day to a single dose whole-body irradiation. Twenty-four hour after irradiation, blood samples were collected from the retroorbital plexus and serum was separated. Afterwards, rats were sacrificed; ovarian tissues were dissected, washed with ice-cold saline, and then, weighed. Serum was separated by centrifugation at $3000 \mathrm{~g}$ for 15 min and kept frozen at $-80^{\circ} \mathrm{C}$ until assessment of $17 \beta$-estradiol (E2) and follicle-stimulating hormone (FSH).

\section{Tissue Collection and Processing:}

Ovarian tissues were homogenized at 1:10 (w:v) in saline ( $\mathrm{pH}$ 7.4) with an Ultra Turrax homogenizer after that the supernatant was obtained by centrifugation at $10,000 \mathrm{~g}$ for $15 \mathrm{~min}$ then, stored at $-80^{\circ} \mathrm{C}$ for biochemical assessment. In addition, further ovarian tissues were fixed in an appropriate buffer for light microscopical examination.

\section{Histopathological Examination}

The ovaries were fixed in 10\% formalin overnight and embedded in paraffin. Serial sections of 4 $\mu \mathrm{m}$ thick were stained with hematoxylin and eosin (H\&E) for light microscopic histological examination. In all ovarian samples, the fifth cut was chosen to count the number of follicles and to evaluate follicular development using a digital video camera mounted on a light microscope (CX21, OLYMPUS, JAPAN).

\section{Circulating Levels of Serum E2 and FSH:}

Serum E2 was assessed using (Monobind, Inc., USA) ELISA kit and FSH were measured using (DRG International, Inc., Springfield) ELISA kit. All procedures were done according to the manufacturer's instructions.

\section{Oxidative Stress Markers}

\section{Reduced glutathione and lipid peroxidation}

GSH and lipid peroxidation were assessed in ovarian supernatant using the commercially available assay kit; GSH assay Kit commercially available from (Biodiagnostic, Egypt). All procedures were carried out according to the manufacturer's instructions. Quantities of ovarian GSH and malondialdehyde were expressed as micromoles and nanomoles per gm weight tissue.

\section{Statistical Analysis}

Data are presented as the mean $\pm \mathrm{SD}$ and were analyzed by one-way analysis of variance (ANOVA) followed by Tukey-Kramer as a post-hoc test. The 0.05 level of probability was used as the criterion for significance. All statistical analyses were performed using Instat version 3 software package. Graphs were sketched using GraphPad Prism (ISIH software, USA) version 5 software.

\section{RESULTS}

\section{Circulating Hormone Levels:}

Female irradiated rats have an undetectable level of serum E2 (74.60 vs. $124.22 \mathrm{pg} / \mathrm{ml}$ in controls) as shown in (Fig.1A) while increased serum FSH levels (20.44 \pm 0.94 vs. $14.216 \pm 1.83 \mathrm{ng} / \mathrm{ml}$ in controls) reaching 1.4 fold as compared with the control group (Fig. 1B).

On the other hand, the serum E2 levels was significantly increased in rats treated with cetrorelix before irradiation by 1.8 folds, respectively when compared with that of the irradiated group (Fig.1A). In contrast, the serum FSH level significantly decreased in cetrorelix-irradiated rats by $60 \%$ as compared with the irradiated group. Furthermore, cetrorelix alone did not show any significant difference in serum E2 and FSH levels as compared to the control group (Fig. 1B). 

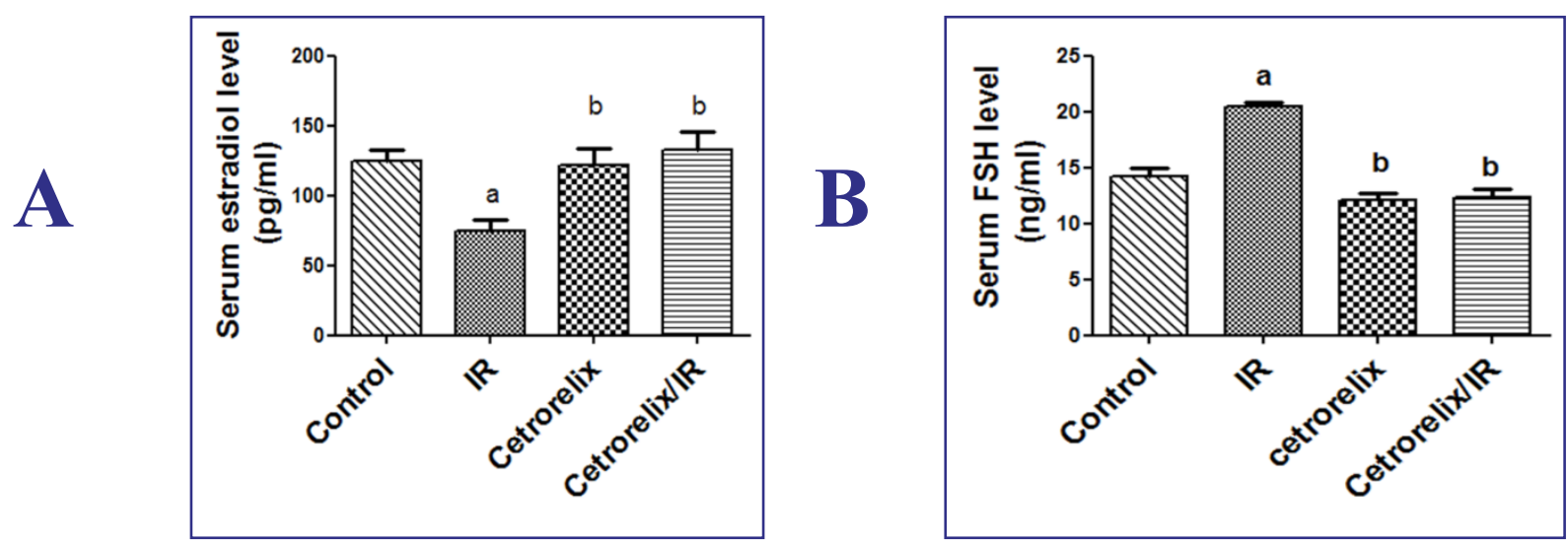

Fig. (1): Changes in Serum E2 (A). and FSH (B) following cetrorelix treatment $(0.5 \mathrm{mg} / \mathrm{kg}$ once daily for 10 days) in rats subjected to $\mathrm{\gamma}$-irradiation (IR).

Data are given as mean, \pm SD for groups. a or b, Statistically significant from the control or radiation group, respectively, at $\mathrm{P}$ $\leq 0.05$ using one-way ANOVA followed by Tukey-Kramer as a post hoc test.

\section{Markers of Folliculogenesis}

Ovarian sections from the control group stained with hematoxylin and eosin (H\&E) showed a normal ovarian structure which was characterized by different types of follicles, oocytes, and corpus luteum (Ph. 1A). No abnormal histological alterations were observed in ovarian sections obtained from animals treated with cetrorelix alone as compared to the control group (Ph. 1C). In irradiated ovaries, the stromal
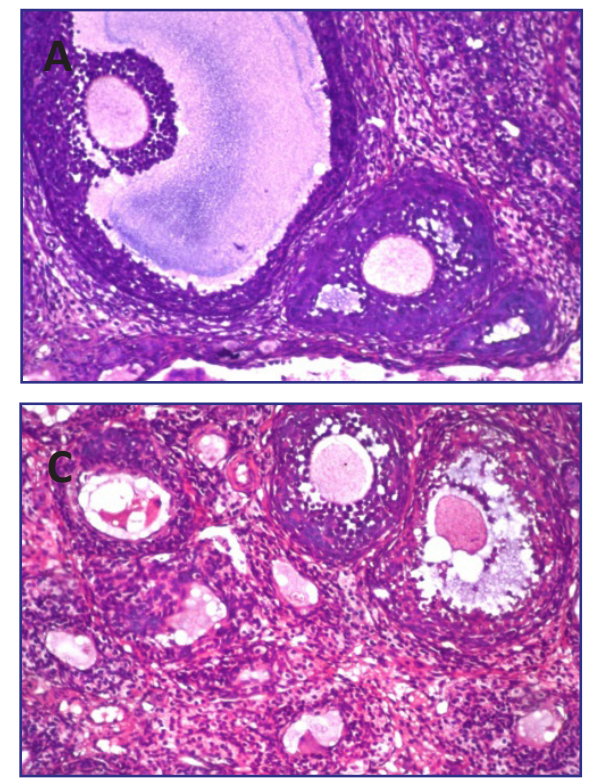

tissue occupied most of the ovarian structure (Ph. 1B), which was characterized by severe hemorrhage and decreasing the number of growing follicles. In addition, very few primordial follicles were detected, and no primordial follicle stock was observed at the periphery of the irradiated ovary (Ph. 1B). Administration of cetrorelix before irradiation preserved ovarian tissue from radiation-induced hemorrhage and maintained the follicular stock (Ph. 1D).
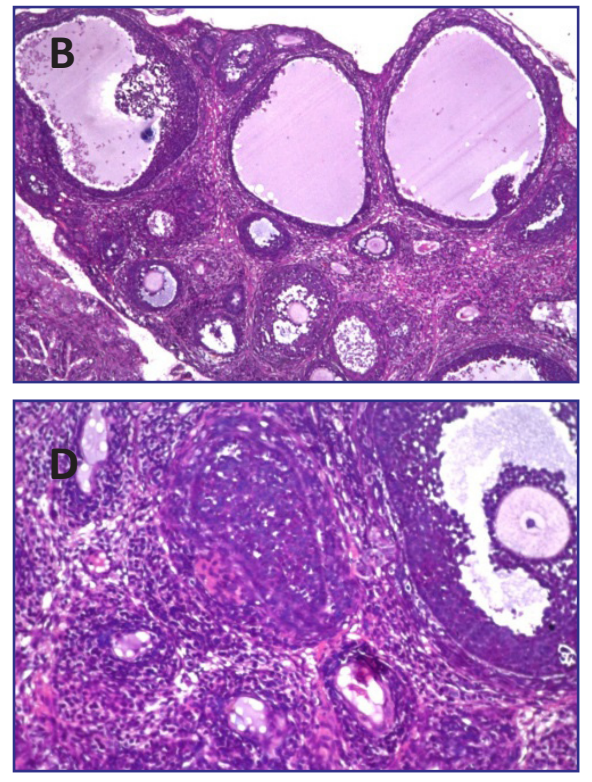

Photo 1. Representative histopathological examination of ovarian tissue sections In which control ovaries represented in A, irradiated ovaries in B ,cetrorelix treated ovaries C and irradiated ovaries pre-treated with cetrorelix D. Histological sections showed that control and and cetrorelix treated ovaries exhibit similar organization. Compared with controls, $\mathrm{\gamma}$-irradiated ovaries have few, if any, resting oocytes in the cortex with severe hemorrhage . Many oocytes in small primary follicles are degenerating in irradiated ovaries. 
Morphometric analysis, during the immature period, showed that irradiated ovaries displayed a sequential reduction in the number of certain classes of follicles. The population of primordial follicles was reduced by $58 \%$ in irradiated ovaries as compared to control( $11.6 \pm 1.5170$ vs. $27.8 \pm 2.59$ in controls), (Fig. 2A). Additionally, radiation reduced the number of preantral follicles by $59 \%$ as compared to control ( $10.0 \pm 4.743$ vs. $24.4 \pm 4.45$ in controls), (Fig. 2B). In addition, there was significant decrease by $55 \%$ in the population of healthy antral follicles between control and irradiated ovaries $(11.4 \pm 1.67$ vs. 25.4 \pm 3.21 in control,) (Fig. 2C). Conversely, the number of atretic follicles increased significantly in irradiated ovaries by $300 \%$ as compared to control (54.4 \pm 3.05 vs.13.6 \pm 2.302 in controls), (Fig. 2D). Treatment of animals with cetrorelix alone showed no significant effect on the follicles' population as compared to the control (Fig. 2B).
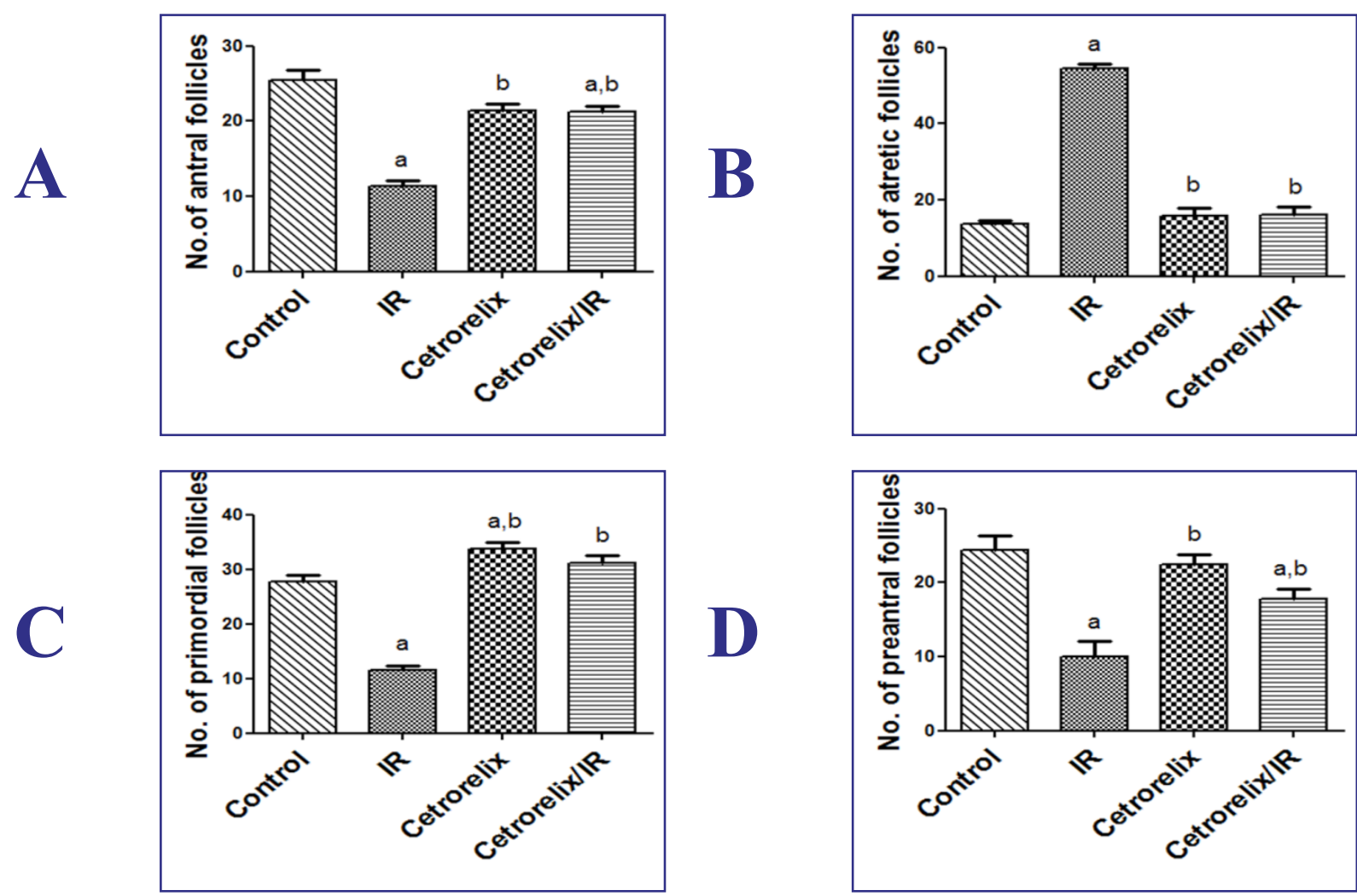

Fig. (2): Morphometric Analysis of Ovarian Follicles Populations. (A) primordial follicles, (B) preantral, (C) antral and (D) atretic. Bars represent the mean $\pm \mathrm{SD}$ of at least three independent experiments. a or b: Statistically significant from control or irradiated group, respectively at $\mathrm{P} \leq 0.05$. Data were analyzed by one-way ANOVA followed by Tukey-Kramer as a posthoc test.

\section{Oxidative Stress Markers}

Oxidative stress markers induced by radiation was assessed in rat ovaries by determination of GSH and lipid peroxides levels. As shown in (Fig. 3), irradiation resulted in a marked GSH depletion by $69 \%$ as compared to control (17.68 \pm 5.25 vs. $57.36 \pm 10.23$ in controls) (Fig. 3A). Additionally, irradiation induced a marked elevation in lipid peroxide levels by $167 \%$ as compared to control value (Fig. 3B). Interestingly, administration of cetrorelix significantly attenuated the irradiation-induced lipid peroxidation and improved the ovarian GSH content as well as the anti-oxidant enzymes activities. 

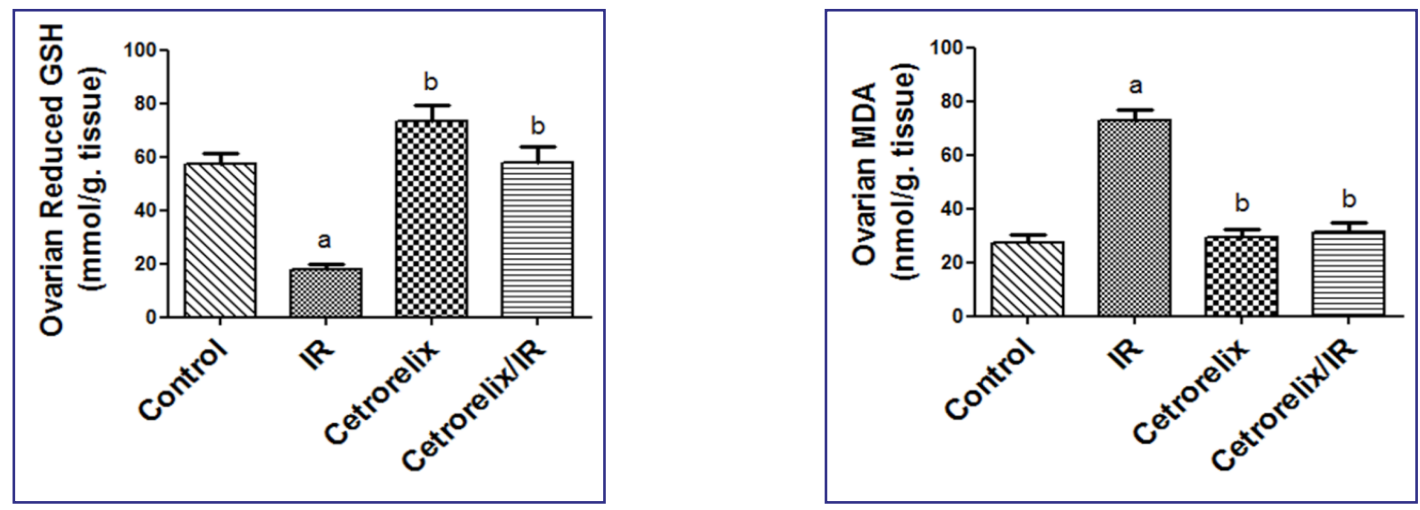

Fig. (3): Effect of cetrorelix on oxidative stress markers in rats subjected to a single dose whole-body irradiation (3.2 Gy). Reduced glutathione (A),malonaldehyde (MDA) (B), Data are given as mean, \pm SD for groups. a or b, Statistically significant from the control or radiation group, respectively, at $\mathrm{P} \leq 0.05$ using one-way ANOVA followed by Tukey-Kramer as a post hoc test.

\section{DISCUSSION}

The significant improvement of cancer treatments entailed a longer life in cancer survivors and raised expectations for higher quality of life with minimized long-term toxicity. However, premature ovarian failure occurs in $31 \%$ of adult female survivors of childhood cancer (Vassilakopoulou et al., 2016). There is significant interest in developing strategies to protect the ovaries from damage during radiotherapy. Interestingly, the most widely studied approach uses gonadotropin releasing hormone agonists/antagonists, but most of them are still under investigation (Thomakos et al., 2015). Previously, the use of GnRH antagonist during chemotherapy was potentially supported, where they may exert a protective effect on ovarian function with an acceptable side effect profile (Whitehead et al., 2011). However, its potential in-vivo radioprotective effect on the female reproductive organs is still uncertain. In the present study, the molecular mechanisms underlying the potential ovarian radioprotective effect of cetrorelix has been assessed by studying its effects on different markers of oxidative stress and folliculogenesis in an experimental model of radiation-induced ovarian failure.

In the irradiated rats, serum E2 was decreased whereas FSH was drastically increased when com- pared with the control value; findings reflect the presence of typical premature ovarian failure (POF) (Lutchman Singh et al., 2005). The exact mechanism of action of $\mathrm{GnRH}$ antagonists in protecting the ovary from radiotherapy is not yet known; they are thought to work by combination of central suppression of gonadotropins at the level of the pituitary, blocking of gonadotropin receptors in the ovary (Murase et al., 2005). The increase in FSH level was attributed to abnormal steroid secretion from the ovaries. Pre-treatment of female rats with cetrorelix prevented the decrease in E2 level and the increase in FSH level induced by irradiation. Our results are in accordance with the previous studies which showed that cetrorelix supplementation decreased FSH levels and resulted in E2 level near the basal level in rat model of chemotherapy-induced ovarian failure (Whitehead et al., 2011). A clinical study found that lowering elevated serum FSH by cetrorelix could raise E2 level and induce pregnancy finally (Check and Katsoff, 2008) .

Indeed, ovarian follicles are the functional units within the female gonad (Trombly et al., 2009) and the female fertility is tightly limited by the size of the ovarian primordial follicle stock whose formation is initiated during fetal life and is completed at or shortly after birth (Skinner, 2005). In the present study, $24 \mathrm{hr}$ after irradiation of females, there was a 
depletion of oocytes contained in primordial follicles so, demonstrating POF (Said et al., 2012) . Besides primordial follicles' depletion, irradiation induced a depletion of the growing pre-antral and antral follicles within a time period of 24-h. Our results confirmed the previous study of the protective effect of cetrorelix on ovarian follicle population induced to damage by chemotherapy through increased primordial follicles stock (Meirow et al., 2004). Of interest, the injection of cetrorelix increased the number of preovulatory follicles, reflecting the augmentation also observed in ovarian weight, suggesting that this compound improves follicular development in radiation induced POF model (Parborell et al., 2005). In the present study, it was found that two weeks after exposure to $\gamma$-radiation resulted in a significant increase in ovarian lipid peroxides accompanied with a significant depletion of GSH levels as compared with the control group. Our result showed that cetrorelix has some mechanisms by which improve follicular development is through decreasing lipid peroxidation, which contradicted with study of (Celik $\boldsymbol{e t}$ al., 2012) . Curiously, the current study demonstrated that cetrorelix treatment preserves the primordial follicles stock and minimizes the follicle depletion induced by radiation.

\section{REFERENCES}

- Adriaens, I.; Smitz, J.; and Jacquet, P. (2009): The current knowledge on radiosensitivity of ovarian follicle development stages. Hum. Reprod. Update, 15: 359.

- Celik, E.; Celik, O.; Kumbak, B.; Yilmaz, E.; Turkcuoglu, I.; Simsek, Y.; Karaer, A.; Minareci, Y.; Ozerol, E.; and Tanbek, K. (2012): A comparative study on oxidative and antioxidative markers of serum and follicular fluid in GnRH agonist and antagonist cycles. J. Assist. Reprod. Genet., 29: 1175.

- Check, J.H.; and Katsoff, B. (2008): Ovulation induction and pregnancy in a woman with premature menopause following gonadotropin suppression with the gonadotropin releasing hormone antagonist, cetrorelix--a case report. Clin. Exp. Obstet. Gynecol., 35: 10

- Gosden, R.G.; Wade, J.C.; Fraser, H.M.; Sandow, J.; and Faddy, M.J. (1997): Impact of congenital or experimental hypogonadotrophism on the radiation sensitivity of the mouse ovary. Hum. Reprod., 12: 2483.

- Gurgan, T.;Salman, C.; and Demirol, A. (2008): Pregnancy and assisted reproduction techniques in men and women after cancer treatment. Placenta, 29: Suppl. B., 152.

- Huirne, J.A.; Homburg, R.; and Lambalk, C. B. (2007): Are GnRH antagonists comparable to agonists for use in IVF? Hum. Reprod., 22: 2805.

- $\quad$ Li, X.; Kang, X.; Deng, Q.; Cai, J.; and Wang, Z. (2013): Combination of a GnRH agonist with an antagonist prevents flare-up effects and protects primordial ovarian follicles in the rat ovary from cisplatininduced toxicity: a controlled experimental animal study.RB. \& E., 11: 16.

- Lutchman Singh, K.; Davies, M.; and Chatterjee, R. (2005): Fertility in female cancer survivors: pathophysiology, preservation and the role of ovarian reserve testing. Hum. Reprod. Update, 11: 69.

- Meirow, D.; Assad, G.; Dor, J.; and Rabinovici, J. (2004): The GnRH antagonist cetrorelix reduces cyclophosphamide-induced ovarian follicular destruction in mice. Hum. Reprod., 19: 1294.

- Murase, M.; Uemura, T.; Gao, M.; Inada, M.; Funabashi, T.; and Hirahara, F. (2005): GnRH antagonist-induced down-regulation of the mRNA expression of pituitary receptors: comparisons with GnRH agonist effects. Endocr. J., 52: 131.

- $\quad$ Parborell, F.; Irusta, G.; Vitale, A.; Gonzalez, O.; Pecci, A.; and Tesone, M. (2005): Gonadotropin-releasing hormone antagonist antide inhibits apoptosis of preovulatory follicle cells in rat ovary. Biol. Reprod., 72: 659. 
- Said, R.S.; El-Demerdash, E.; Nada, A.S.; and Kamal, M. M. (2016): Resveratrol inhibits inflammatory signaling implicated in ionizing radiation-induced premature ovarian failure through antagonistic crosstalk between silencing information regulator 1 (SIRT1) and poly(ADP-ribose) polymerase 1 (PARP1). Biochem. Pharmacol., 103: 140.

- Said, R.S.; Nada, A.S.; and El-Demerdash, E. (2012): Sodium selenite improves folliculogenesis in radiation-induced ovarian failure: a mechanistic approach. PloS one, 7: 50928.

- Skinner, M.K. (2005). Regulation of primordial follicle assembly and development. Hum. Reprod. Update, 11: 461.

- Stroud, J. S.; Mutch, D.; Rader, J.; Powell, M.; Thaker, P.H.; and Grigsby, P.W. (2009): Effects of cancer treatment on ovarian function. Fertil. Steril., 92: 417.

- Thomakos, N.; Trachana, S. P.; Koutroumpa, I.; Rodolakis, A.; and Gavalas, N.G. (2015): Molecu- lar aspects and clinical methods for preserving ovarian reserves in women receiving cancer treatment. Clin. Exp. Obstet. Gynecol., 42: 416.

- Trombly, D. J.; Woodruff, T. K.; and Mayo, K. E. (2009). Suppression of Notch signaling in the neonatal mouse ovary decreases primordial follicle formation. Endocrin., 150: 1014.

- Vassilakopoulou, M.; Boostandoost, E.; Papaxoinis, G.; de La Motte Rouge, T.; Khayat, D.; and Psyrri, A. (2016): Anticancer treatment and fertility: Effect of therapeutic modalities on reproductive system and functions. Critical reviews in oncol./hemat., 97: 328 .

- Whitehead, J.; Toledo, M.G.; and Stern, C.J. (2011): A pilot study to assess the use of the gonadotrophin antagonist cetrorelix in preserving ovarian function during chemotherapy. The Australian \& New Zealand J. Obstet. Gynaecol., 51: 452. 